\title{
Modeling the Large Scale Structures of Astrophysical Jets in the Magnetically Dominated Limit
}

\author{
Hui $\mathrm{Li}^{1}$, Giovanni Lapenta ${ }^{2}$, John M. Finn ${ }^{2}$, Shengtai $\mathrm{Li}^{3}$, and Stirling A. Colgate ${ }^{4}$
}

\begin{abstract}
We suggest a new approach that could be used for modeling both the large scale behavior of astrophysical jets and the magnetically dominated explosions in astrophysics. We describe a method for modeling the injection of magnetic fields and their subsequent evolution in a regime where the free energy is magnetically dominated. The injected magnetic fields, along with their associated currents, have both poloidal and toroidal components, and they are not force free. The dynamic expansion driven by the Lorentz force of the injected fields is studied using 3-dimensional ideal magnetohydrodynamic simulations. The generic behavior of magnetic field expansion, the interactions with the background medium, and the dependence on various parameters are investigated.
\end{abstract}

Subject headings: magnetic fields — galaxies: active — galaxies: jets — methods: numerical - MHD

\section{INTRODUCTION}

It is generally thought that energies (including magnetic fields) in jets and lobes of radio galaxies observed on large scales (from kpc to Mpc) are supplied from the central black hole accretion region, despite our poor understanding on how exactly this has occurred. Near the black hole, studies have focused on the initial formation of jets, in terms of jet collimation and acceleration. Here, both hydromagnetic limit (e.g., Blandford \& Payne 1982; Ouyed \& Pudritz 1997; Ustyugova et al. 1999; and others) and Poynting flux limit (e.g., Blandford 1976; Lovelace 1976; Lynden-Bell 1996; Ustyugova et al. 2000; Li et al. 2001; Lovelace et al.

\footnotetext{
1 Applied Physics Division, MS B227, Los Alamos National Laboratory, NM 87545; hli@lanl.gov

2 Theoretical Division, MS K717, Los Alamos National Laboratory, NM 87545; lapenta@lanl.gov; finn@lanl.gov

${ }^{3}$ Theoretical Division, MS B284, Los Alamos National Laboratory, NM 87545; sli@lanl.gov

${ }^{4}$ Theoretical Division, MS B227, Los Alamos National Laboratory, NM 87545; colgate@lanl.gov
} 
2002; and others) have been investigated, with various degree of success [see Ferrari (1998) for a relatively recent review].

On much larger scales, jet propagation, morphologies (such as lobe formation, hot-spots, filaments, etc.) and associated instabilities have been investigated extensively. In particular, much progress has been made in the so-called kinetic energy dominated regime, for both non-relativistic and relativistic flows. The pioneering work by Norman et al. (1988) set the stage for nearly two decades of studies on how highly supersonic, light (jet material density less than the surrounding density), and sometimes magnetized, collimated flows propagate in various (uniform and/or stratified) background medium (e.g., Norman et al. 1983; Martí et al. 1994; Clarke 1996; Bodo et al. 1998; Tregillis et al. 2004; and many others). Even though magnetic fields have been actively included in some of these studies, the overall behavior has been predominantly determined in the hydrodynamic limit (but see a recent paper by Nakamura \& Meier 2004 in both hydro and Poynting dominated limits).

The relative importance of kinetic energy and magnetic energy in jets and radio lobes has been a subject for extensive studies and debates. Better radio observations on large, $>$ tens of kpc, scales (e.g., Owen et al. 2000) and recent X-ray observations of radio lobes of Fanaroff-Riley type II (FR II) sources (e.g., Croston et al. 2005) have suggested that magnetic energies inside the radio lobes are significant (see also Kronberg et al. 2001). These observations bring out the questions whether jets/lobes could be magnetically dominated and what are the physical processes govern such flows on these large scales. The studies on this Poynting flux dominated limit in large scales have been relatively sparse.

The purpose of the present paper is to present an approach to model the large scale magnetic structure generated by a purely magnetic energy input. We describe a method of injecting magnetic fields into a small volume and following the subsequent evolution of the injected magnetic free energy. This free energy causes magnetic fields to expand under their own Lorentz force. This expansion is subsequently affected by interacting with the background environments. We believe that this approach could in principle be applied to different astrophysical systems. Here, we concentrate on its applications to modeling the large scale morphologies of radio jets and lobes. In $\S 2$, we describe our basic assumptions and approach on how to model magnetic field injection. Numerical methods used in our simulations are also discussed. In $\S 3$, we present the simulation results on the evolution of magnetic fields for different parameters and setups. In $\S 4$, we summarize our results. 


\section{Basic Approach and Model Assumptions}

Since it is currently impossible to simulate the whole process from the jet formation near the black hole accretion disk out to radio lobes because of the vast length scale separation, we adopt the following general framework which separates the jet/lobe structure into different regions: I: the engine region, where the black hole accretion disk system produces magnetic fields and these fields are ejected above and below the disk. II: the propagation region, where energy, mass, and magnetic fields flow through the background medium. III: the termination region, where these flows are significantly slowed down or come to a stop. In our approach, for region I, we emphasize that we are not modeling the black hole accretion disk system and how magnetic fields are ejected from such systems. Instead, we attempt to model the likely outcome of the energy/mass supply from the black hole accretion system as having particular functional forms in non-force-free magnetic fields, along with certain injection rates. In this sense, we are focusing on the energy/mass flows in an external medium, and how they would eventually terminate (i.e. Regions II and III). In particular, we study the limit that the injected free energy is predominantly in magnetic fields, which is different from the previous approach where the flow is dominated by the kinetic energy (i.e., the injected kinetic energy is much larger than the injected magnetic and thermal energy). In the following sections, we will discuss the functional forms of injected magnetic fields and the numerical setups for modeling the nonlinear evolution of these magnetic fields.

\subsection{Basic Equations, Magnetic Fields, Mass Injections and Numerical Codes}

We solve the ideal MHD equations in three-dimensional Cartesian coordinate system $\{x, y, z\}$ :

$$
\begin{aligned}
\frac{\partial \rho}{\partial t}+\nabla \cdot(\rho \mathbf{v}) & =\dot{\rho}_{\mathrm{inj}} \\
\frac{\partial(\rho \mathbf{v})}{\partial t}+\nabla \cdot\left(\rho \mathbf{v} \mathbf{v}+P_{\mathrm{g}}+P_{\mathrm{B}}-\mathbf{B B}\right) & =0 \\
\frac{\partial E}{\partial t}+\nabla \cdot\left[\left(E+P_{\mathrm{g}}+P_{\mathrm{B}}\right) \mathbf{v}-\mathbf{B}(\mathbf{v} \cdot \mathbf{B})\right] & =\dot{E}_{\mathrm{inj}} \\
\frac{\partial \mathbf{B}}{\partial t}-\nabla \times(\mathbf{v} \times \mathbf{B}) & =\dot{\mathbf{B}}_{\mathrm{inj}}
\end{aligned}
$$

where all variables have their usual meaning. We use an ideal gas equation of state with an adiabatic index $\gamma=5 / 3$, which relates the internal energy density $e$ and the gas pressure $P_{\mathrm{g}}$ as $e=P_{\mathrm{g}} /(\gamma-1)$. The magnetic energy density is expressed as $P_{\mathrm{B}}=B^{2} / 2$. The total energy $E=\rho v^{2} / 2+P_{\mathrm{g}}+P_{\mathrm{B}}$. We have neglected external forces such as gravity in these 
simulations. This is not correct for some astrophysical environments such as jets in galaxy clusters. The main focus of this paper is to present the salient features of this new approach, and we will present more detailed astrophysical applications in future publications.

We have added an injection term $\dot{\mathbf{B}}_{\text {inj }}$ in the induction equation to incorporate the external injection of magnetic fields $\left(\nabla \cdot \dot{\mathbf{B}}_{\mathrm{inj}}=0\right)$. The injection process is modeled as:

$$
\dot{\mathbf{B}}_{\mathrm{inj}}=\left\{\begin{array}{cc}
\gamma_{b} \mathbf{B}_{\mathrm{inj}} & \text { for } t \leq t_{\mathrm{inj}} \\
0 & \text { for } t>t_{\mathrm{inj}}
\end{array}\right.
$$

where $\mathbf{B}_{\text {inj }}$ describes the magnetic fields whose exact form is given in the following section. The $\gamma_{b}(t)$ specifies the injection rate for magnetic fields, and it is usually taken as a constant over a finite time internal $t \leq t_{\mathrm{inj}}$ and as zero (e.g., the injection is turned off) for $t>t_{\mathrm{inj}}$. More general forms of injection can be easily included as well.

In addition, we have included mass injection in the central region, motivated by the possibility that matter can be ejected together with magnetic fields away from the central source. Note that both the magnetic field and mass injections violate the ideal MHD condition, in the sense that the total mass on magnetic field lines are allowed to change. Furthermore, adding mass unto field lines helps to alleviate the numerical problems of having extreme low density regions after magnetic fields have undergone large expansions. Without the detailed knowledge on how and what amount of mass could get unto the field lines, we adopt the following simple formula:

$$
\dot{\rho}_{\text {inj }}=\gamma_{\rho} \rho_{0} \exp \left[-\left(r^{2}+z^{2}\right) / r_{\rho}^{2}\right],
$$

where $r_{\rho}$ and $\gamma_{\rho}$ are the characteristic radius and rate for mass injection. The injected mass is further assumed to have the same velocity and temperature as that of the gas in the injection region.

Furthermore, there is an energy injection along with the magnetic field and mass injections. The energy injection rate from the magnetic fields injection can be expressed as

$$
\dot{E}_{\mathrm{inj}}=\mathbf{B} \cdot \dot{\mathbf{B}}_{\mathrm{inj}}=\gamma_{b} \mathbf{B}_{\mathrm{inj}} \cdot \mathbf{B},
$$

while in general both $\mathbf{B}$ and $\mathbf{B}_{\text {inj }}$ have temporal and spatial dependences. Note that there is a small amount of thermal and kinetic energy injected, accompanied with the mass injection. But this energy is negligibly small compared with the injected magnetic energy.

All simulations are performed using a new ideal MHD package developed at Los Alamos (Li \& Li 2003). This package uses high-order Godunov-type finite volume numerical methods. These methods conservatively update the zone-averaged fluid and magnetic field quantities based on estimated advective fluxes of mass, momentum, energy and magnetic field at zone 
interfaces. Due to the discontinuities (contacts and shocks) in the solutions, Roe's Riemann solver with an entropy-fix (Powell et al. 1994) is used to calculate these fluxes. It usually solves the total energy equation as the ideal MHD equations are written into the conservative form. To maintain pressure positivity in regions where the pressure can become very small or negative, we also solve two auxiliary equations: the internal energy equation and the modified entropy equation,

$$
\begin{aligned}
\frac{\partial e}{\partial t}+\nabla \cdot(e \mathbf{v}) & =-P_{\mathrm{g}} \nabla \cdot \mathbf{v} \\
\frac{\partial S}{\partial t}+\nabla \cdot(S \mathbf{v}) & =0
\end{aligned}
$$

where $S=P_{\mathrm{g}} / \rho^{(\gamma-1)}$ is defined as the modified entropy. The exact usage of these two equations is described in Ryu et al. (1993) and Balsara \& Spicer (1999a).

The divergence free condition of the magnetic field $\nabla \cdot \mathbf{B}=0$ is ensured by a constrained transport (CT) scheme flux-CT (Balsara \& Spicer 1999b). The CT scheme can yield magnetic fields that are different from the updated values based on the Godunov method. The difference may be large enough to lead to negative pressure. To minimize the change in energy conservation, we update the total energy with the newly-calculated magnetic fields only when the pressure becomes negative. The whole package is parallelized via message-passing interface (MPI). A typical run made on parallel Linux clusters with 64 processors takes 3 hrs.

\subsection{Injected Magnetic Field Configuration}

We determine the injected magnetic fields using three key quantities: the length scale of the injection region (which we designate as $r=1$ ), the amount of poloidal flux $\left(\Psi_{p}\right)$, and the poloidal current $\left(I_{z}\right)$. For simplicity, we also assume that the injected magnetic fields $\mathbf{B}_{\text {inj }}$ are axisymmetric. In cylindrical coordinates $\{r, z, \phi\}$, we specify the injected poloidal flux function $\Psi$ as, (where $\Psi=r A_{\phi}$ and $A_{\phi}$ is the $\phi$ component of vector potential),

$$
\Psi(r, z)=r^{2} \exp \left(-r^{2}-z^{2}\right)
$$

up to a normalization coefficient $B_{0}$. Note that one can easily introduce another scale variable in the $z$-direction which separates the scaling between radial and vertical directions. Here, we have taken them to be same for simplicity. In the global distributions, we envision that the poloidal flux and current will point along one direction in one region (say, for $r \leq 1$ ) and "return" in the opposite direction in another region (say, for $r>1$ ), so the total net poloidal flux and current are zero. 
The above-mentioned key ingredients are motivated by the general framework of treating the black hole accretion disk system as a dynamo "machine", from which we use Eq.(10) as a simple representation of the axisymmetric part of the poloidal flux that might come from the dynamo process. The continuous shearing of the poloidal flux lines by the disk's differential rotation generates the toroidal magnetic fields which correspond to a large scale poloidal current. Although the system scale we are modeling here is much larger than the actual size of the black hole accretion disk system, we envision that there still exists such global poloidal flux and current distributions on these scales.

The poloidal fields, up to a normalization coefficient $B_{0}$, are:

$$
\begin{aligned}
B_{\mathrm{inj}, r} & =-\frac{1}{r} \frac{\partial \Psi}{\partial z}=2 z r \exp \left(-r^{2}-z^{2}\right) \\
B_{\mathrm{inj}, z} & =\frac{1}{r} \frac{\partial \Psi}{\partial r}=2\left(1-r^{2}\right) \exp \left(-r^{2}-z^{2}\right)
\end{aligned}
$$

The poloidal fields have an $O$-point at $(r, z)=(1,0)$ where both $B_{\mathrm{inj}, r}$ and $B_{\mathrm{inj}, z}$ are zero. In specifying the toroidal component $B_{\mathrm{inj}, \phi}$, we adopt the approach of writing

$$
B_{\mathrm{inj}, \phi}=f(\Psi) / r
$$

where $f(\Psi)$ is an arbitrary function of $\Psi$. Physically, this means that we have made the $\hat{\phi}$-component of the Lorentz force $(\mathbf{J} \times \mathbf{B})_{\phi}$ of the injected magnetic fields equal to zero. However, this does not prevent rotation being developed during the evolution by the Lorentz force of the combined fields and currents from both the injected fields and the already existing fields. Specifically, we choose $f(\Psi)=\alpha \Psi$, which means

$$
B_{\mathrm{inj}, \phi}=\alpha \Psi / r=\alpha r \exp \left(-r^{2}-z^{2}\right),
$$

where $\alpha$ is a constant and it has the units of inverse length scale. Other forms of $f(\Psi)$ are certainly possible, which will give different pitch profiles for the magnetic fields.

Fig. 1. - left: Shown is the radial component of the $\mathbf{J} \times \mathbf{B}$ force as a function of radius $r$ in the equatorial plane with $\alpha=1,3$, and 5 for solid, dot-dashed and dashed lines, respectively. right: Shown is the vertical component of the $\mathbf{J} \times \mathbf{B}$ force as a function of radius $r$ at the $z=0.5$ plane with $\alpha=1,3$, and 5 for solid, dot-dashed and dashed lines, respectively.

This simple initial configuration has several properties. Firstly, the magnetic fields are "localized" because the flux and energy drop exponentially as a function of distance from the center. This is an important difference from many previous studies where large scale background magnetic fields are often assumed to be present initially. In the poloidal plane, this global dipole-like configuration gives zero net flux and current. 
Secondly, the parameter $\alpha$ roughly specifies the flux ratio between toroidal and poloidal components. The poloidal flux between $0 \leq r \leq 1$ is

$$
\Phi_{p}=2 \pi \Psi(1,0)=2 \pi / e \approx 2.3
$$

and the toroidal flux threading through the $\{x, z\}$ plane with $x \geq 0$ is

$$
\Phi_{t}=\alpha \sqrt{\pi} / 2 \approx 0.9 \alpha
$$

So, the poloidal and toroidal fluxes are roughly equal when $\alpha \approx 2.6$. In astrophysical jets powered by AGNs, it is likely that magnetic fields are highly wound up by the disk rotation, this will imply that $\alpha>>1$. The poloidal current flowing through the middle plane $(z=0)$ with $0 \leq r \leq 1$ is

$$
I_{z}=2 \pi f(\Psi(1,0))=2 \pi \alpha / e \approx 2.3 \alpha .
$$

The total toroidal current is

$$
I_{\phi}=\iint J_{\phi} d r d z=2 \sqrt{\pi}
$$

The ratio of poloidal $\left(I_{z}\right)$ to toroidal current $\left(I_{\phi}\right)$ is $\alpha \sqrt{\pi} / e \approx 0.65 \alpha$, i.e., the poloidal current becomes dominant when $\alpha \gg 1$.

Thirdly, it is important to realize that this initial field is not in force equilibrium because $\mathbf{J} \times \mathbf{B} \neq 0$. The current density $\mathbf{J}=\nabla \times \mathbf{B}_{\text {inj }}$ is given as

$$
\begin{aligned}
& J_{r}=2 \alpha z r \exp \left(-r^{2}-z^{2}\right) \\
& J_{z}=2 \alpha\left(1-r^{2}\right) \exp \left(-r^{2}-z^{2}\right) \\
& J_{\phi}=2 r\left(5-2 z^{2}-2 r^{2}\right) \exp \left(-r^{2}-z^{2}\right) .
\end{aligned}
$$

Along the equatorial plane $(z=0)$, the radial force density is given as

$$
F_{r}(z=0)=2 r\left(1-r^{2}\right)\left(10-\alpha^{2}-4 r^{2}\right) \exp \left(-2 r^{2}\right) .
$$

It can be seen that at large $r, F_{r}>0$, meaning that the magnetic fields always expand outward due to the "hoop" forces. At small $r$ and small $\alpha$, the force is radially outward as well, whereas for small $r$ but large $\alpha$, the force is radially inward (i.e., "pinching"). Figure 1 (left) illustrates these effects along the equatorial plane for different $\alpha$ values. Along the vertical direction,

$$
F_{z}=2 z r^{2}\left(\alpha^{2}-10+4 z^{2}+4 r^{2}\right) \exp \left(-2 r^{2}-2 z^{2}\right)
$$


For $\alpha^{2}>10, F_{z}$ is always positive (negative) for $z>0(z<0)$, driving the fields away from the mid-plane. For smaller $\alpha$, fields at large spherical radii will still expand away from the origin. Figure 1 (right) shows the vertical force as a function of $r$ at $z=0.5$ for different $\alpha$.

For the sake of completeness, we also give the total magnetic energy of this field:

$$
E_{0}=\int\left(B_{\mathrm{inj}}^{2} / 2\right) d V=\sqrt{\frac{\pi}{2}} \frac{\left(5+\alpha^{2}\right) \pi}{8} \approx 0.5\left(5+\alpha^{2}\right)
$$

out of which the first and second terms are from the poloidal and toroidal magnetic field components, respectively.

In summary, we have devised a magnetic field structure that is governed by the poloidal flux $\Psi$ and poloidal current $I_{z}$. This magnetic field is localized in space, containing both poloidal and toroidal fluxes which are interwoven (i.e., finite helicity), and will dynamically evolve (i.e., not in force-free equilibrium), especially along the vertical direction in the large $\alpha$ limit. As we will show later in detail, it is this vertical expansion along the symmetry axis that forms the basis of modeling the large scale structure of jets.

\subsection{Units for $3 \mathrm{D}$ Simulations}

The initial magnetic field structure is embedded in a plasma background with finite gas pressure and density, both of which are taken to be uniform for simplicity in this study. We take $P_{\mathrm{g}}=P_{0}, \rho=\rho_{0}$, and the sound speed $c_{s}=\sqrt{\gamma P_{\mathrm{g}} / \rho} \approx 1.3 \sqrt{P_{0} / \rho_{0}}$. The magnetic field strength normalization parameter is $B_{0}$. Simulations are performed in the Cartesian geometry with a uniform mesh. The simulation domain is a cube and the resolution is usually taken as $240 \times 240 \times 240$ or higher.

For the following runs, we typically choose $\rho_{0}=1, P_{0}=1$, and $B_{0}=1$. To put these numbers in an astrophysics context: suppose that the code unit $\rho_{0}=1$ corresponds to a background density of $3 \times 10^{-3}$ particles $/ \mathrm{cm}^{3}$, and the background temperature is $7 \mathrm{keV}$. This means that the sound speed in the code unit $c_{s}=\sqrt{\gamma} \approx 1.3$ corresponds to $1.1 \times 10^{8}$ $\mathrm{cm} / \mathrm{s}$. The combined density and temperature relation also means that the magnetic field $B_{0}=1$ in the code units corresponds to a physical magnetic field of $\sim 20 \mu \mathrm{G}$. Suppose the code unit $L=1$ corresponds to $15 \mathrm{kpc}$, then the simulation duration $t=5$ corresponds to $\sim 9.2 \times 10^{7} \mathrm{yrs}$, and a total energy of 200 corresponds to $\sim 6.2 \times 10^{59}$ ergs. The poloidal flux as given in Eq.(15) is $\sim 9.4 \times 10^{40} \mathrm{Gcm}^{2}$, and the poloidal current as given in Eq.(17) is $\sim 1.7 \alpha \times 10^{18}$ Ampere. 


\section{Results}

We present the simulation results for two cases: the first has impulsively "injected" magnetic fields (i.e., as initial conditions) for $\alpha=20$. The second case is with a continuous injection of magnetic fields.

Fig. 2.- The impulsive injection case with $\alpha=20$. (left): Shown is the azimuthally averaged pressure in the $\{r, z\}$ plane. Azimuthally averaged poloidal flux surfaces (white-color) are overlaid as well. They are at $t=0,0.5,1$, respectively. (right): Same as in the left panel, except that $t=3,4,5$, respectively. Note the cartesian computational box is $[-12,12]$ in all three directions for the simulation, and axis limits and colorbars have been changed between left and right panels.

\subsection{Impulsive Injection with an initial $\alpha=20$}

We choose $P_{0}=\rho_{0}=B_{0}=1$ and $\alpha=20$ for this run and the initial $\mathbf{B}$ is of the form of $\mathbf{B}_{\text {inj. }}$. The injection coefficients $\gamma_{b}$ and $\gamma_{\rho}$ are taken to be zero (i.e., no continuous injection of $\mathbf{B}$ or $\rho$ ). The computational box is taken to be $[-12,12]$ in all three directions. These parameters give the maximum initial magnetic field $|B| \approx 8.6$ at $(r, z) \approx(0.7,0)$.

Fig. 3. - The impulsive injection case with $\alpha=20$. Shown is the overall energy evolution of different components. The magnetic, kinetic and changes in thermal energies are shown as solid, dashed, dot-dashed curves, respectively. The total energy is shown by the thin solid curve at the top. As the system evolves, the initial magnetic energy is converted to both the thermal energy and the kinetic energy of the plasma.

The "free" energy of the system is initially all magnetic. Since the initial magnetic field is out of force equilibrium (see Figure 1), evolution is expected. Because the evolution remains quite axisymmetric (see below), in Figure 2, we show the time sequence of azimuthally averaged pressure distribution, overlaid with azimuthally averaged poloidal flux function $\Psi$. In Figure 3, we show the overall energy evolution for different components. The initial magnetic energy is given by Eq.(24) with $\alpha=20$. The kinetic energy is $\rho v^{2} / 2$ integrated over the whole volume, and the change in thermal energy is $\left[P_{\mathrm{g}}(t)-P_{\mathrm{g}}(0)\right] /(\gamma-1)$ integrated over the whole volume, where $P_{\mathrm{g}}(t)$ and $P_{\mathrm{g}}(0)$ are the pressure distributions at time $t$ and $t=0$.

The whole evolution can be approximately separated into two stages. The first stage $(0<t<1)$ is a rapid conversion of $\sim 70 \%$ of the available magnetic energy through the 
work done by the $\mathbf{J} \times \mathbf{B}$ force on the surrounding plasmas, which pinches all the flux lines within $r=1$ radially inward and expands flux lines vertically away from the mid-plane. Since $\alpha$ in this case is quite high, the $\mathbf{J} \times \mathbf{B}$ force is consequently so strong that it initially drives both a radially converging shock and an axially expanding shock, as indicated by the central red region and the red region surrounding the magnetic fields in the left panel of Figure 2. These shocks heat the plasmas efficiently and generate large amounts of entropy. The second stage is much slower, represented by the slow dissipation of magnetic energy and further gradual heating of the plasma. The magnetic field expansion gradually slows down. In the meantime, a compressional shock wave, shown as the outer red region in the right panel of Figure 2, continues with its expansion. Figures 4 and 5 show the time evolution of the azimuthally averaged radial profiles of the radial velocity $v_{r}$ and the plasma pressure $P_{\mathrm{g}}$. A shock front, generated from the initial impulsive release of the magnetic energy, can indeed be seen. This shock heats the plasmas surrounding the magnetic structure as indicated by the propagating pressure front. The continued expansion, however, also makes a central, low pressure cavity. Note that the gas pressure changes from this wave propagation are quite strong $(\sim 20-40 \%)$.

Fig. 4. - The impulsive injection case with $\alpha=20$. Shown is the radial distribution of the azimuthally averaged (cylindrical) radial velocity $v_{r}$ at $t=0.5,1.5, \ldots, 4.5$, respectively. The profiles are taken at $z=2.4$. The front generated by the sudden release of magnetic energy is moving supersonically.

Fig. 5. - The impulsive injection case with $\alpha=20$. Shown is the radial distribution of the azimuthally averaged pressure at $t=0.5,1.5, \ldots, 4.5$, respectively. The profiles are taken at $z=2.4$. At early time, the "pinching" of inner magnetic fields greatly increases the pressure near the central axis. This is followed by expansion which creates a central low pressure (and density) region. The front is moving supersonically.

Fig. 6. - The impulsive injection case with $\alpha=20$. Shown is the magnetic fields $|B|$, density and pressure distributions in the $\{x, z\}$ plane $(y=0)$ at $t=5$. The overall structure remains axisymmetric. The magnetic field expansion creates a central low density and low pressure region.

Figure 6 shows the distribution of the magnitude of magnetic fields $|B|$, density $\rho$ and gas pressure $P_{\mathrm{g}}$ at $t=5$ in the $\{x, z\}$ plane $(y=0)$. These distributions suggest that the final state is still quite axisymmetric. The magnetic fields form an elongated structure due to both the pinch at the central region and the vertical expansion along the symmetry axis. 
Fig. 7. - The impulsive injection case with $\alpha=20$. Shown is one magnetic field line at $t=5$. The field line goes up in a tightly wound central helix and comes back in a loosely wound helix.

Fig. 8. - The impulsive injection case with $\alpha=20$. (Upper): The radial distribution of magnetic field components $B_{z}$ and $B_{\phi}$. (Lower): The radial distribution of the plasma pressure $P_{g}$, the magnetic pressure $P_{B}$, and the total pressure $P_{\text {tot }}=P_{g}+P_{B}$. Both panels are taken at $z=3$ and $t=5$.

Figure 7 depicts a 3D field line, showing its going up in a central tightly wound helix and coming down outside in a loosely wound helix. Based on the rate of field twists, one might expect that this helix should be kink unstable. The hollow pressure profile as shown in Figure 6, however, may be a stabilizing influence.

Figure 8 shows the radial distribution of azimuthally averaged magnetic field components (top) and different pressure components (bottom) at $z=3$ and $t=5$. The expansion of the magnetic fields from an initial sphere with a radius of $\sim 1$ to the elongated structure have created a very low density cavity since plasmas are tied to the expanding magnetic fields. The pressure within this cavity is mostly from the shock heating. The cavity is dominated by magnetic pressure (the plasma $\beta=P_{\mathrm{g}} / P_{B}$ is $\sim 10 \%$ near the axis). A careful force balance analysis shows that the central region is nearly force free (more detailed results will be presented elsewhere). Going to large radii $(r \sim 1-1.5)$, however, the structure is confined by the gas pressure, which prevents further expansion of the magnetic fields.

Since the density is quite low inside the cavity, there is some numerical heating, which amounts to an error of $\sim 2 \%$ increase in the total energy (the top thin solid curve in Figure 3 ). Some of the poloidal flux lines at late times (the plot at $t=5$ of Figure 2) indicate numerical magnetic reconnection at the mid-plane.

\subsection{Continuous Injection}

Fig. 9.- The continuous injection case with an injection $\alpha=15$. Shown is the evolution of azimuthally averaged poloidal flux lines during and after the magnetic field injection. Different plots are for $t=0,2.5,4,5.5$, and 7 . The injection is terminated at $t=2.5$. Each subplot has ten, evenly spaced contour lines.

For this case, we adopt the functional form of $\mathbf{B}_{\text {inj }}$ for both the initial and injected 
magnetic fields. For the initial fields, we choose $P_{0}=\rho_{0}=1, B_{0}=1$, and $\alpha=3$. For the continuous field injection, we use $B_{0}=0.2, \alpha=15, \gamma_{b}=3, \gamma_{\rho}=0.1, r_{\rho}=0.5$, and $t_{\text {inj }}=2.5$. The computational box is $[-12,12]$ in all three dimensions. This run uses a grid of $320^{3}$. To put these numbers in an astrophysics context, using the conversions given in the beginning of this section, we have: the simulation's $t_{\text {inj }}=2.5$ corresponds to $\sim 4.6 \times 10^{7} \mathrm{yrs}$, and a total energy of 200 corresponds to $\sim 6.2 \times 10^{59} \mathrm{ergs}$. The mass injection rate 0.1 corresponds to $\sim 1 M_{\odot} / y r$ and the magnetic field $B_{0}=0.2$ corresponds to $\sim 4 \mu \mathrm{G}$. All these parameters are roughly consistent with the physical conditions in the central region of galaxy clusters. However, note that the background medium is not stratified in the current simulation, so the total thermal energy within a computational volume of $10^{3}$ would correspond to $\sim 3 \times 10^{60}$ ergs. In other words, the magnetic fields are expanding into a volume with a high thermal pressure background.

Figure 9 shows the evolution of azimuthally averaged poloidal flux lines in the $\{r, z\}$ plane. The initial poloidal flux has a maximum of $\sim 0.37$, then the poloidal flux increases to $\sim$ 0.9 at $t=2.5$ when the injection finishes. Magnetic fields continue to expand somewhat after the injection has stopped. Figure 10 shows the energy evolution for different components. The injected energy is essentially all in magnetic fields, with a very small amount in the form of thermal and kinetic energies of the injected plasmas associated with $\dot{\rho}_{\text {inj }}$. There is again a little numerical heating at the late time (see the top two curves in Figure 10) due to the existence of extremely low density regions.

Part of the injected magnetic energy is gradually converted into both the kinetic energy and the changes in the thermal energy of the surrounding plasmas. The system is approaching a quasi steady state with very slow or no changes in different energy components. We interpret this as that the injected magnetic fields run out of their "drive" since the magnetic field injection stops at $t=2.5$. Subsequently, the magnetic fields have come to be in rough force balance with the surroundings. Of course the whole system can not be steady, it is still expanding, though very slowly. Figure 11 shows the distribution of magnetic fields, density and gas pressure at $t=7$. A magnetically dominated cavity is evident.

Figure 12 shows the radial distribution of azimuthally averaged magnetic field components and pressures at $t=7$ and $z=3$. Again, we have a central column which is

Fig. 10.- The continuous injection case with an injection $\alpha=15$. Shown is the evolution of different energy components. The thicker solid, dashed and dot-dashed lines are for magnetic, kinetic and changes in thermal energies, respectively. The thinner dashed and solid curves at the top are for the total injected energy and the total energy in the computational box, respectively. 
magnetically dominated, with nearly the force-free condition. At a larger radius $(r \sim 1.5)$, a small gas pressure gradient is present to balance the residual magnetic pressure gradient. This hollow pressure profile may be helpful to stabilize the whole structure.

It is interesting to note that more than half of the injected magnetic energy still remains in the system for this set of parameters. Compared with the impulsive injection case with $\alpha=20$, the two systems have about the same initial "free" energy, and we can see that the continuous injection case retains a much larger fraction of the magnetic energy and gives a slightly larger magnetically dominated volume.

\section{Discussions}

In this paper, we have presented a method of injecting free energy in the purely magnetic form. The non-force-free nature of these magnetic fields causes expansion in a self-pinched, collimated fashion. In relating to astrophysical jets, we assume that the whole radio galaxy can be divided into: a central region where the black hole accretion system resides, a propagation region which contains the jets and outflows supplied by the central region, and a termination region where the lobes are formed. Based on this assumption, we suggest that the approach taken here could be used to study both the propagation and termination regions. We have especially concentrated on the magnetically dominated regime, different from the previous studies where energy is predominantly carried by the kinetic energy of the flow.

The 3D MHD simulation results for a few relatively simple cases are presented here, mostly for the purpose of illustrating the salient features of the particular functional form of the injected magnetic fields and how the subsequent evolution might be related to it. As such, we have only explored a limited set of parameters and their influence on the magnetic field evolution. Many important issues are not addressed here but will be presented in forthcoming papers. For example, it will be important to know the stability of such magnetic structures. The field line plots show that they are highly wounded, suggesting kink unstable, yet the final distributions seem to suggest that they remain axisymmetric and stable. The hollow pressure profile might have a stabilizing influence, but more detailed studies are required in order to

Fig. 11. - The continuous injection case with an injection $\alpha=15$. Shown is the magnetic field, density and pressure distributions (from left to right) at $t=7$. The central low density and pressure volume is occupied with and dominated by magnetic fields. A compressional shock wave generated during the magnetic field injection stage has propagated away from the central, magnetized region. 
draw firm conclusions on the stability. In addition, how background environment can affect the propagation speed and general morphologies, how the final configuration depends on the injection rates, and what determines the total amount of magnetic energy dissipation, etc. It is thus premature to compare the current simulation results directly with observations of radio jets/lobes, though this is our ultimate goal.

HL acknowledges useful discussions with D. Ryu and M. Nakamura. This research was performed under the auspices of the Department of Energy. It was supported by the Laboratory Directed Research and Development Program at LANL and by IGPP at LANL.

\section{REFERENCES}

Balsara, D.S., \& Spicer, D.S. 1999a, J. Comput. Phys., 148, 133

Balsara, D.S., \& Spicer, D.S. 1999b, J. Comput. Phys., 149, 270

Blandford, R.D. 1976, MNRAS, 176, 465

Blandford, R.D. \& Payne, D.G. 1982, MNRAS, 199, 883

Bodo, G. et al. 1998, A\&A, 333, 1117

Clarke, D.A. 1996, in Energy Transport in Radio Galaxies and Quasars, eds. P.E. Hardee, A.H. Bridle, J.A. Zensus, ASP Conf. Ser. 100, p.311, San Francisco: Astron. Soc. Pac.

Croston, J.H. et al. 2005, ApJ, 626, 733

Ferrari, A. 1998, ARAA, 36, 539

Kronberg, P.P. et al. 2001, ApJ, 560, 178

Li, S., \& Li, H. 2003, Technical Report, LA-UR-03-8935, Los Alamos National Laboratory

Li, H. et al. 2001, ApJ, 561, 915

Fig. 12. - The continuous injection case with an injection $\alpha=15$. (Upper): The radial distribution of azimuthally averaged magnetic field components $B_{z}$ and $B_{\phi}$. (Lower): The radial distribution of the plasma pressure $P_{g}$, the magnetic pressure $P_{B}$, and the total pressure $P_{\text {tot }}=P_{g}+P_{B}$. Both panels are taken at $z=3$ and $t=7$. 
Lovelace, R.V.E. 1976, Nature, 262, 649

Lovelace, R.V.E. et al. 2002, ApJ, 572, 445

Lynden-Bell, D. 1996, MNRAS, 279, 389

Martí, J.Ma., et al. 1994, A\&A, 281, L9

Nakamura, M., \& Meier, D.L. 2004, ApJ, 617, 123

Norman, M.L., Winkler, K-H.A., \& Smarr, L. 1983, in Astrophysical Jets, eds. A. Ferrari, A.G. Pacholczyk, p227, Dordrecht: Reidel

Norman, M.L., Burns, J.O., \& Sulkanen, M.E. 1988, Nature, 335, 146

Ouyed, R., \& Pudritz, R.E. 1997, ApJ, 482, 712

Owen, F. et al. 2000, ApJ, 543, 611

Powell, K.G. et al. 1999, J. Comput. Phys., 154, 284

Ryu, D. et al. 1993, ApJ, 414, 1

Tregillis, I.L., Jones, T.W., \& Ryu, D. 2004, ApJ, 601, 778

Ustyugova, G.V. et al. 1999, 516, 221 
This figure "f1.jpg" is available in "jpg" format from: http://arxiv.org/ps/astro-ph/0604469v1 
This figure "f2a.jpg" is available in "jpg" format from: http://arxiv.org/ps/astro-ph/0604469v1 
This figure "f2b.jpg" is available in "jpg" format from: http://arxiv.org/ps/astro-ph/0604469v1 
This figure "f3.jpg" is available in "jpg" format from: http://arxiv.org/ps/astro-ph/0604469v1 
This figure "f4.jpg" is available in "jpg" format from: http://arxiv.org/ps/astro-ph/0604469v1 
This figure "f5.jpg" is available in "jpg" format from: http://arxiv.org/ps/astro-ph/0604469v1 
This figure "f6.jpg" is available in "jpg" format from: http://arxiv.org/ps/astro-ph/0604469v1 
This figure "f7.jpg" is available in "jpg" format from: http://arxiv.org/ps/astro-ph/0604469v1 
This figure "f8.jpg" is available in "jpg" format from: http://arxiv.org/ps/astro-ph/0604469v1 
This figure "f9.jpg" is available in "jpg" format from: http://arxiv.org/ps/astro-ph/0604469v1 
This figure "f10.jpg" is available in "jpg" format from: http://arxiv.org/ps/astro-ph/0604469v1 
This figure "f11.jpg" is available in "jpg" format from: http://arxiv.org/ps/astro-ph/0604469v1 
This figure "f12.jpg" is available in "jpg" format from: http://arxiv.org/ps/astro-ph/0604469v1 